\title{
Research Progress on Electromagnetic Textile Materials
}

\author{
Jianying Chen ${ }^{1}$ and Hong Xiao ${ }^{2 *}$ \\ ${ }^{1}$ College of Textiles, Donghua University, China \\ ${ }^{2}$ Institute of Quartermaster Engineering \& Technology, China
}

*Corresponding author: Xiao Hong, Institute of Quartermaster Engineering \& Technology, Institute of System Engineering, Academy of Military Science, PLA, Beijing, China.

Received Date: March 05, 2019

Published Date: March 14, 2019

\section{Abstract \\ The electromagnetic parameter of common textile materials has not reached the order of magnitude of metals or semiconductors [1]. They generally do not own any electromagnetic function. In the article, the concept of electromagnetic textile material and its basic preparation method are proposed. Finally, the application of electromagnetic textile materials is briefly introduced [2].}

Keywords: Textile materials; Electromagnetic function; Preparation; Application

\section{Introduction}

Common textile materials have dielectric properties and electrostatic phenomena, but the electromagnetic parameter of them has not reached the order of magnitude of metals or semiconductors. The electromagnetic properties of textile materials include electrical conductivity [3], dielectric properties, electrostatic and magnetic properties, etc. Therefore, they generally do not own any electromagnetic function.

With the rapid development of conductive polymer in-situ polymerization technology, metal, metal oxide and fiber material hybrid technology and the metallization processing technology of fiber materials, the conductor preparation technology of textiles has become more and more perfect, forming a series of electromagnetic functional electromagnetic textile materials such as shielding textiles, antistatic textiles, absorbing materials, wearable antennas etc. [4].

\section{Electromagnetic Textile Materials}

Electrical conduction is the movement of electrically charged particles through a transmission medium. Conductive property is determined by the type of atoms in a material. Materials in which a large number of electrons can move freely are called conductors, and Materials with low electron mobility are called insulators.
Ordinary fibers are generally made of non-conductive and nonmagnetic polymer materials. Electromagnetic textile materials are a new type of functional textile materials obtained from fibers or yarns with good electrical and magnetic properties through textile processing technology or coating conductive material on the common textile material in the finishing process. Meanwhile, electromagnetic textile materials have unique structure of textile materials and the electromagnetic properties of the metal materials [5].

\section{Preparation of Electromagnetic Materials}

To obtain the functionalization of textile materials, special materials must be introduced during the preparation process. Textile materials include fibers, yarns and fabrics. Therefore, electromagnetic functionalization of fibers, yarns and fabrics can be achieved in spinning, weaving and finishing [6] .

In the spinning process for fibers, metal fibers, carbon/graphite fibers or intrinsically conductive polymer materials having intrinsic electromagnetic function may be used to take place of the ordinary fiber materials in whole or in part; It is possible to add the powder having electromagnetic properties to the spinning solution in the blending way during the spinning process [7] (Figure1\&2). 


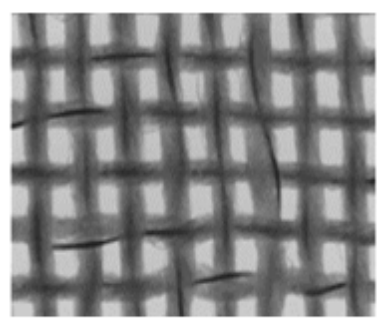

(a) The structure of the fabric

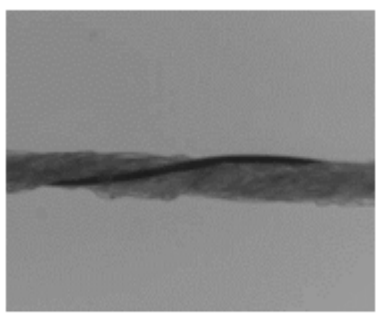

(b) The structure of the fibers

Figure 1: Woven fabrics made of hybrid yarns containing metal wire.

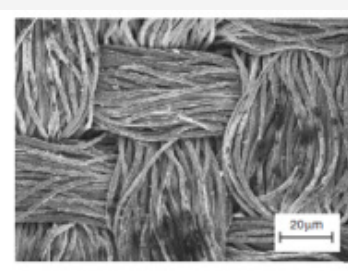

(a) The structure of the fabric

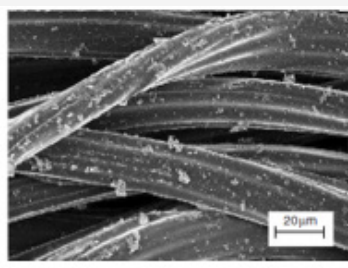

(b) The structure of the fibers

Figure 2: Scanning electron micrpgraphs of PPy coated polyester.

In the spinning process for yarns, electromagnetic fibers such as metal fibers and magnetic fibers can be added to the ordinary fibers through different ways to combine, producing the electromagnetic yarn [8]. Metal fibers have low elongation and poor toughness, so they are not suitable to be used alone for weaving. They are often used to form the yarn containing metal fiber with ordinary textile fibers by blending, enveloping, etc.

In the weaving process, electromagnetically functionalized yarns can be directly woven. The common yarns can be interlaced into fabrics with the electromagnetically functionalized yarns [9].

The finishing process are suitable for fibers, yarns and fabrics [10]. For the fiber or the yarn that has been formed and does not have electromagnetic function, the surface of it may be coated with a metal coating or magnetic powder by electroless plating, electroplating, magnetron sputtering or other ways. For ordinary fabrics without electromagnet properties, the surface can be treated by finishing, such as the electroplating, electroless plating or embroidery to make it electromagnetic [11].

\section{The Application of Electromagnetic Textile Materials}

\section{Antistatic textile materials}

The surfaces of materials exhibiting a low surface conductivity tend to charge under certain conditions, especially in a dry environment. The electrostatic phenomenon of cellulose fibers in the processing process is not obvious; but the electrostatic interference of protein fibers is pretty serious. Although the wool fiber has high equilibrium moisture regain, its mass specific resistance is the highest in the natural fiber. The resistivity of synthetic fibers such as polyester, nylon, acrylic, polypropylene, etc., which are generally high in moisture regain, is as high as $10^{14} \Omega \cdot \mathrm{cm}$, and the accumulation of electrostatic charge is obvious (Figure 3).

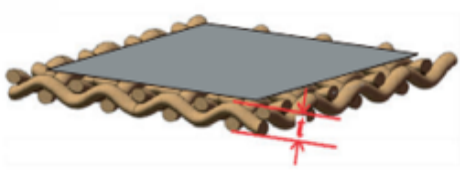

(a) Side view

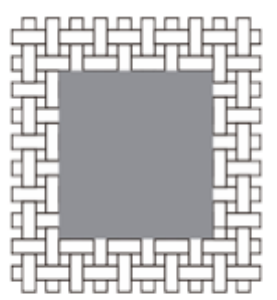

(b) Top view
Figure 3: Schematic diagram of metallized fabric structure.

Antistatic fabric: Making fibers absorb water from the environment, and electrostatic interference in lower spinning is a common method in spinning mills.

In order to prepare relatively durable antistatic fiber, the methods are following.

(1) Adding the surfactant to a fiber-forming polymer during blend spinning;

(2) Adding the hydrophilic group by block copolymerization;

(3) Adding the hydrophilic group by graft modification in a fiber-forming polymer. These can make the fibers obtain durable hygroscopicity and antistatic properties.

In addition, there are also another method, including fixing the surfactant to the surface of the fiber with a binder, or crosslinking the surfactant on the surface of the fiber to form a film. The effect is similar to applying an antistatic varnish onto the surface of the plastic.

Adding a polar group to the surface of the polymer to form a conductive layer or by combining with moisture in the air to form a hydrogen bond can reduce the electrical resistance of the polymer and accelerates the escape of the static charge.

Conductive fabric: The electrical resistivity of the conductive fiber is smaller than that of the antistatic fiber, it has a more significant antistatic effect. And during the blend fabrics with the same antistatic effects the amount of conductive fiber added is much smaller than that of the antistatic fiber. As long as a few thousandths to a few percent of the conductive yarn is added, the fabric can attain antistatic requirements. So, with the widespread use of organic conductive fibers, the field of application of antistatic fibers has been gradually reduced.

For fabrics, it is easy to generate electric charge due to friction among the fibers or contact with other objects. Conductive fiber acting as a good conductor of electricity can dissipate excess electrons through the connection points.

Organic conductive fiber is the most widely used antistatic material in textile engineering. The carbon organic conductive fiber using carbon as a conductive material produced by the composite spinning method is widely used. The organic conductive fiber formed by carbon coating on the outside of the synthetic fiber 
has high conductivity, and the color of organic conductive fibers produced by using metal oxide as conductive materials is close to white. Both of them are also widely applied.

\section{Electromagnetic shielding textile materials}

Electromagnetic shielding is a technical measure to prevent or suppress the transmission of electromagnetic energy by using a shield [12].

Weaving electromagnetic shielding fabric: There are two main types of functional materials for weaving electromagnetic shielding fabrics, metal fibers and metallized fibers. Conductive materials for electromagnetic shielding fabrics are mainly metals and intrinsically conductive polymers [13].

Common conductive fibers are stainless steel, silver, copper, nickel, aluminum, etc. Using textile processing techniques such as weaving, knitting and non-woven, the conductive yarn and the ordinary yarn are intertwined to form a textile shielding material with good shielding effect [14]. The density of these materials is high. And when the higher content of metal yarn is blended with ordinary yarn, the cohesion of the yarn is poor, and it is easy to break.

The surface resistivity of fibers coated by intrinsically conductive polymer like PANi, PPy, and PTh will decrease, and the electromagnetic shielding effectiveness value will improve [15].
However, due to the color limitation of the intrinsically conductive polymer, the textile product has a single color, and the cost is very high.

Metallized electromagnetic shielding fabric: Metallized electromagnetic shielding fabrics are mostly metal-coated fabrics with a metal layer on the surface of the fabric. The surface of the fabric is surface-treated by electroless plating, electroplating, vacuum evaporation to form a metal film, which can improve the shielding effectiveness. However, the metal coating is easy to fall off and is not resistant to washing.

\section{Frequency selective surface textile materials}

Frequency Selective Surface (FSS) is an infinitely large periodic array structure along one-dimensional, two-dimensional, etc. It is mainly divided into two types: patch type and aperture type, which have frequency selective characteristic for the propagation of electromagnetic wave in space. The patch type can totally reflect electromagnetic waves of a specific frequency, and the aperture type can transmit all electromagnetic waves of a specific frequency.

The textiles are light, soft and flexible in processing. Flexible periodic array structure prepared by textile processing technology is called Frequency Selective Fabric (FSF). According to the filtering characteristics, the Frequency Selective Fabric can be divided into four frequency response characteristics: high pass, low pass, band pass and band stop [6] (Figure 4\& Table1).

Table 1: Basic specifications of conductive wire.

\begin{tabular}{|c|c|c|c|c|c|c|}
\hline Number & Specification/(dtex/f) & Substrate & Conductive substance & Color & Section & Manufacturer \\
\hline 1 & $20 / 3$ & PA66 & Carbon & Dark gray & & Solutia \\
\hline 2 & $26.7 / 4$ & PA66 & Carbon & Dark gray & & Solutia \\
\hline 3 & $22.3 / 3$ & PA6 & Carbon & Dark gray & & Konebo \\
\hline 4 & $21.7 / 6$ & PET & Carbon & Black & & Konebo \\
\hline 5 & $22.2 / 3$ & PET & Metal oxide & White & & Konebo \\
\hline 6 & $22.2 / 3$ & PA6 & Metal oxide & White & & Konebo \\
\hline 7 & $27.8 / 3$ & PA6 & Carbon & Light gray & & Teijin \\
\hline 8 & $24.4 / 1$ & PA6 & Carbon & Black & & BASF \\
\hline
\end{tabular}



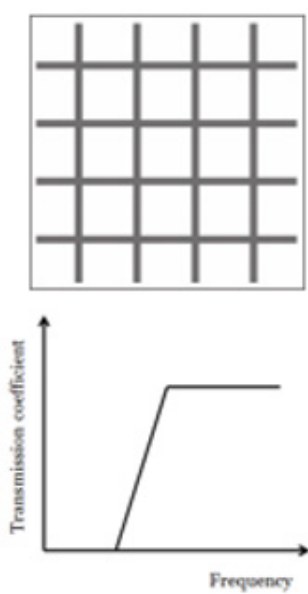

(a) High pass
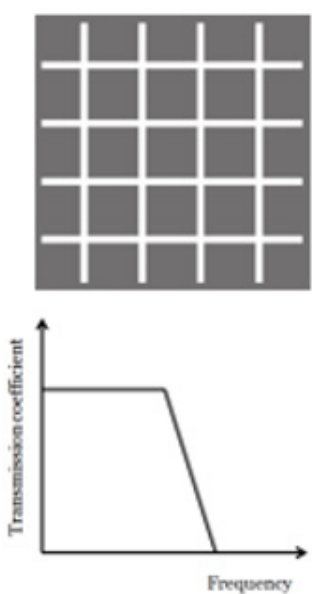

(b) Low pass
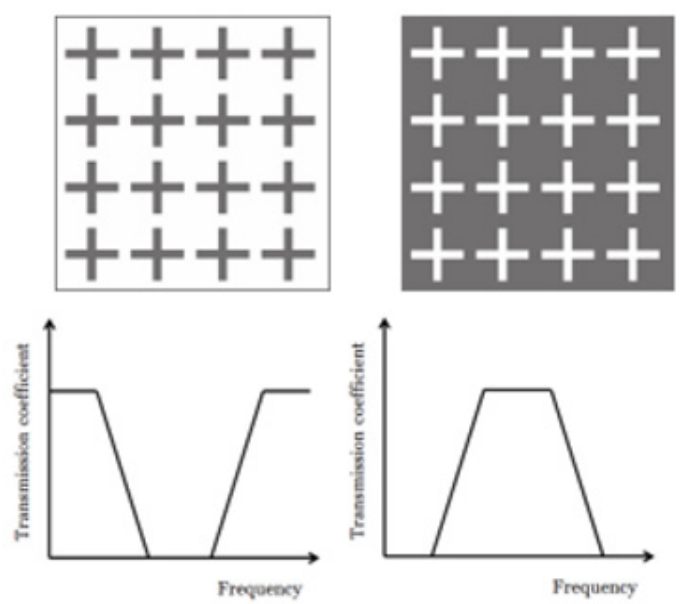

(c) Band stop

(d) Band pass

Figure 4: The four frequency response characteristics of the frequency selective surface.

There are usually several ways to make frequency selective surface textile materials:

(1) The cut commercialized conductive material unit is directly bonded to the non-conductive fabric substrate.

(2) Depositing conductive materials on the surface of fabrics by screen printing, inkjet printing and other textile finishing techniques can form the conductive structural unit [16].

(3) The high conductive yarns are formed into a periodic structural unit by textile weaving processing techniques such as weaving, weft knitting, embroidery and so on $[17,18]$.

\section{Acknowledgement}

The authors disclosed receipt of the following financial support for the research, authorship/or publication of this article: The authors acknowledge support from the National Natural Science Foundation of China (grant number 51673211).

\section{Conflict of Interest}

No conflict of interest.

\section{References}

1. Maity S, Singha K, Debnath P, Singha M (2013) Textiles in electromagnetic radiation protection. Journal of Safety Engineering 2(2): 11-19.

2. Joo J, Epstein AJ (1994) Electromagnetic radiation shielding by intrinsically conducting polymers. Applied Physics Letters 65(18): $2278-2280$

3. Raji RK, Miao X, Boakye A (2017) Electrical conductivity in textile fibers and yarns-review. AATCC Journal of Research 4(3): 8-21.

4. Wang Z, Zhang L, Bayram Y, Volakis JL (2012) Embroidered conductive fibers on polymer composite for conformal antennas. IEEE Transactions on Antennas \& Propagation 60(9): 4141-4147.

5. Radetić M (2013) Functionalization of textile materials with silver nanoparticles. Journal of Photochemistry \& Photobiology C Photochemistry Reviews 48(1): 95-107.
6. Textor T, Mahltig B (2010) A sol-gel-based surface treatment for preparation of water repellent antistatic textiles. Applied Surface Science 256(6): 1668-1674

7. Ozen MS, Sancak E, Beyit A, Usta I, Akalin M (2013) Investigation of electromagnetic shielding properties of needle-punched nonwoven fabrics with stainless steel and polyester fiber. Textile Research Journal 83(8): 849-858.

8. Ortlek HG, Saracoglu OG, Saritas O, Bilgin S (2012) Electromagnetic shielding characteristics of woven fabrics made of hybrid yarns containing metal wire. Fibers \& Polymers 13(1): 63-67.

9. Wang Y, Jing X (2005) Intrinsically conducting polymers for electromagnetic interference shielding. Polymers for Advanced Technologies 16(4): 344-351.

10. Guan F, Hong X, Shi M, Yu W, Wang F (2016) Realization of planar frequency selective fabrics and analysis of transmission characteristics. Textile Research Journal 87(11).

11. Yildiz Z, Usta I, Gungor A (2012) Electrical properties and electromagnetic shielding effectiveness of polyester yarns with polypyrrole deposition. Textile Research Journal 82(20): 2137-2148.

12. Paligová M, Vilčákova J, Sáha $P$, Křesálek V, Stejskal J, et al. (2004) Electromagnetic shielding of epoxy resin composites containing carbon fibers coated with polyaniline base. Physica A Statistical Mechanics \& Its Applications 335(3): 421-429.

13. Dhawan SK, Singh N, Rodrigues D (2003) Electromagnetic shielding behaviour of conducting polyaniline composites. Science \& Technology of Advanced Materials 4(2): 105-113.

14. Kim HK, Kim MS, Song K, Park YH, Kim SH, et al. (2003) EMI shielding intrinsically conducting polymer/PET textile composites. Synthetic Metals 135: 105-106.

15. Locher I, Klemm M, Kirstein T, Trster G (2006) Design and characterization of purely textile patch antennas. IEEE Transactions on Advanced Packaging 29(4): 777-788.

16. Chauraya A, Whittow WG, Vardaxoglou JC, Li Y, Torah R, et al. (2013) Inkjet printed dipole antennas on textiles for wearable communications. IET Microwaves, Antennas \& Propagation 7(9): 760-767.

17. Tennant A, Hurley W, Dias T (2012) Experimental knitted, textile frequency selective surfaces. Electronics Letters 48(22): 1386-1388.

18. Seager RD, Chauraya A, Bowman J, Broughton M, Philpott R, et al. (2013) Fabric based frequency selective surfaces using weaving and screen printing. Electronics Letters 49(24): 1507-1509. 